Supporting Information

\title{
Breathable Nanomesh Humidity Sensor for Real-time Skin Humidity Monitoring
}

Wooseong Jeong, Jinkyu Song, Jihoon Bae, Koteeswara Reddy Nandanapalli, and Sungwon

Lee*

Department of Emerging Materials Science, Daegu Gyeongbuk Institute of Science \& Technology (DGIST), 333, Techno Jungang-daero, Hyeonpung-myeon, Dalseong-gun, Daegu 711-873, Republic of Korea

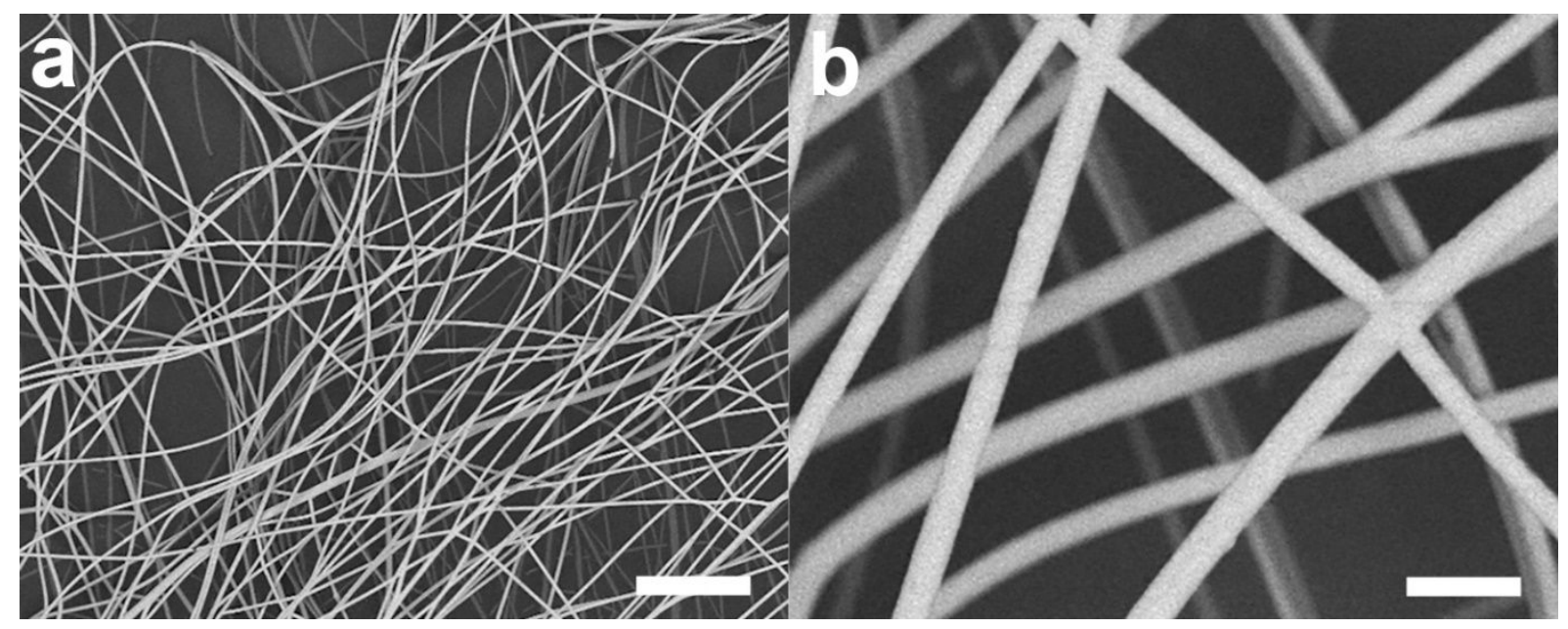

Figure S1. Field-emission scanning electron micrographs of the PVA nanofibers: a) Low resolution $($ scale bar $=15 \mu \mathrm{m})$. b) High resolution $($ scale bar $=2 \mu \mathrm{m})$. 


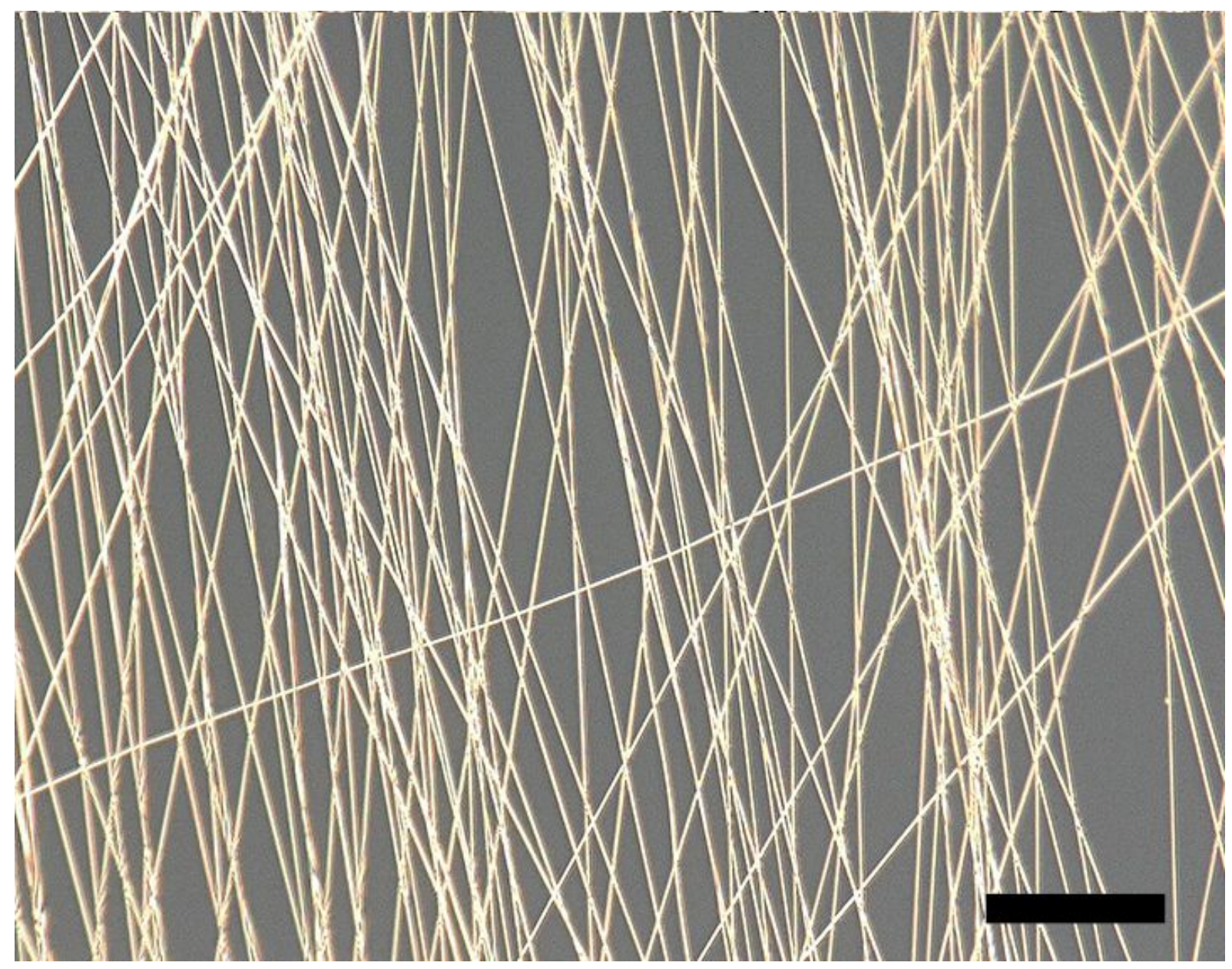

Figure S2. Optical image of the sensor layer that was deposited by gold onto the PVA nanofiber (scale bar $=50 \mu \mathrm{m}$ ). 


\section{Electro Spinning \\ Parylene C Deposition \\ Physical Vapor Deposition}

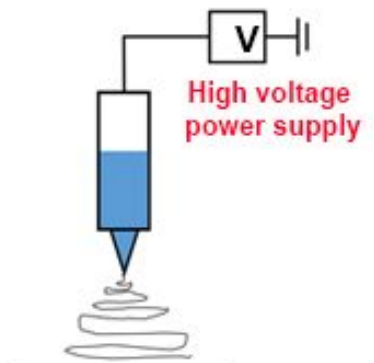

Collector

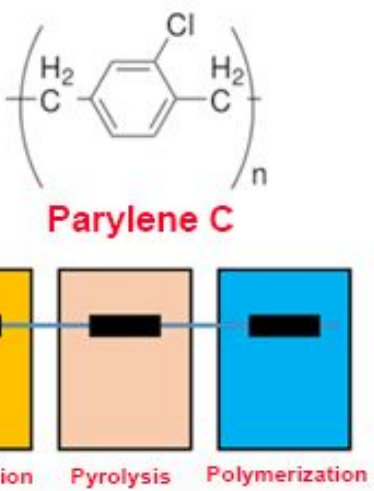

(Au)
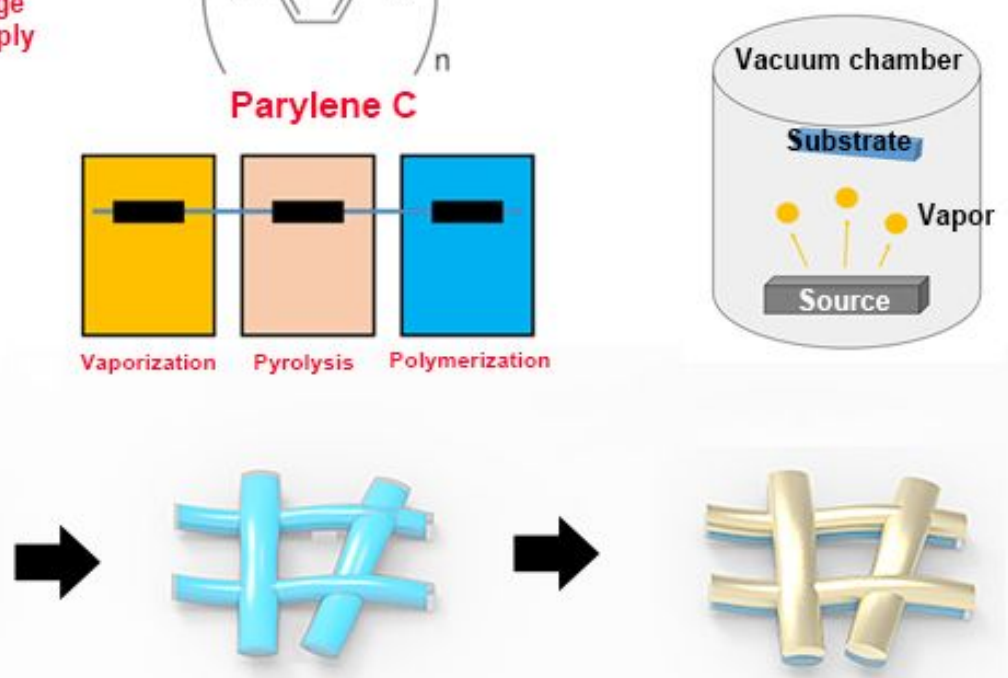

Parylene deposited

Humidity sensor

PVA nanomesh PVA nanomesh

Figure S3. Fabrication method of PVA nanomesh humidity sensor. 


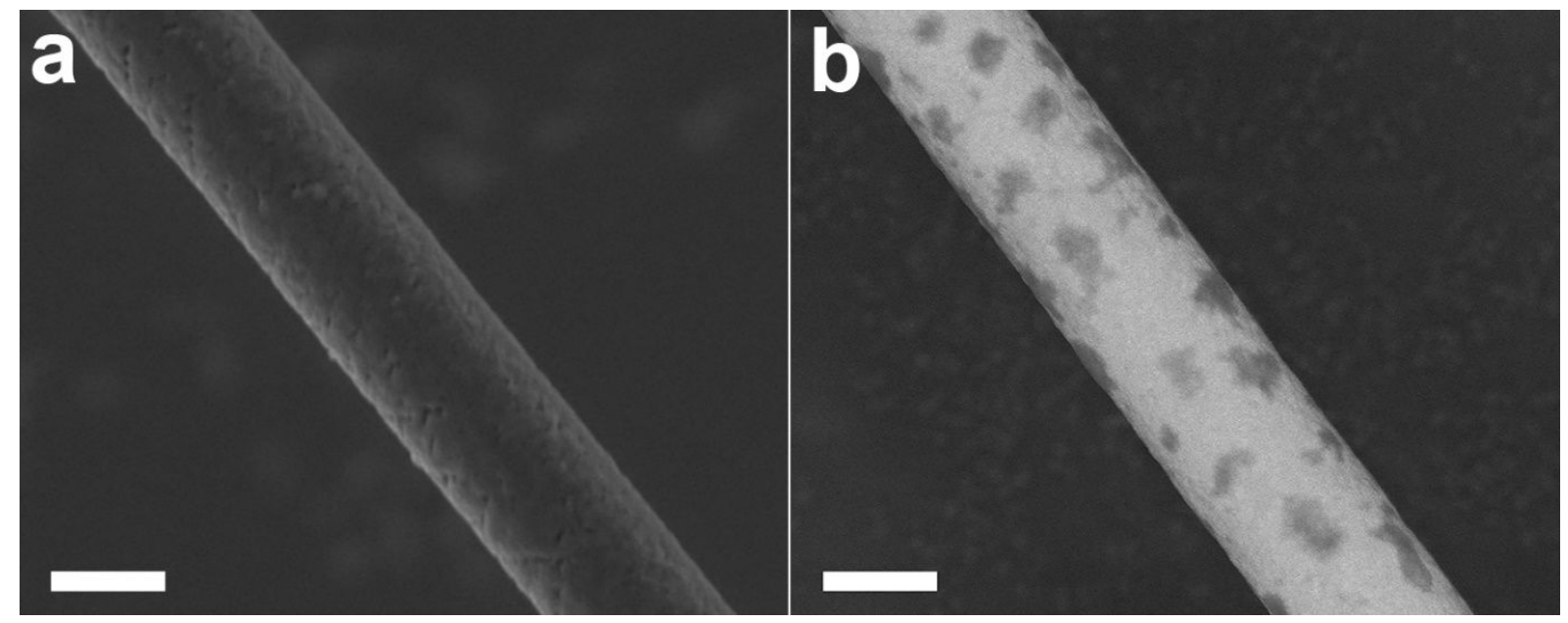

Figure S4 SEM image of a single Parylene C-coated PVA nanofiber (scale bar $=600 \mathrm{~nm}$ ): a) Before and b) after coating with Au (100 nm-thick). 


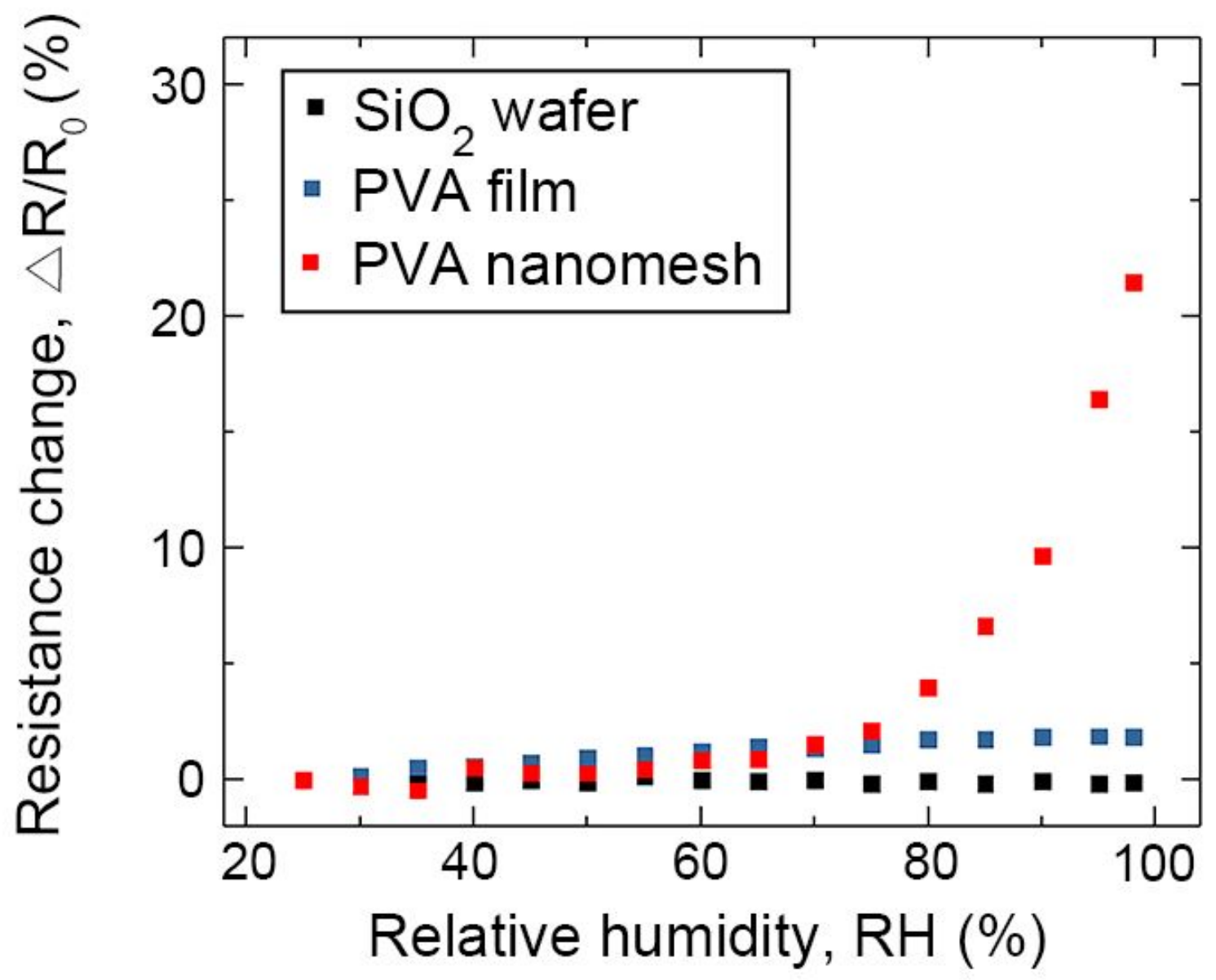

Figure S5. Normalized resistance changes in response to the relative humidity $(\mathrm{RH})$ depending on the substrate.

To analyze the dependence of resistance on $\mathrm{RH}$, the resistance changes were normalized. The variation in the output resistance was calculated as $\Delta \mathrm{R} / R_{0}=\left(R_{\text {final }}-R_{\text {initial }}\right) / R_{\text {initial }}$, where $R_{\text {final }}$ is the resistance at a set RH value, and $R_{\text {initial }}$ is the resistance at $25 \mathrm{RH} \%$. Although the same gold was deposited, sensing capability can vary depending on the substrate geometry. 

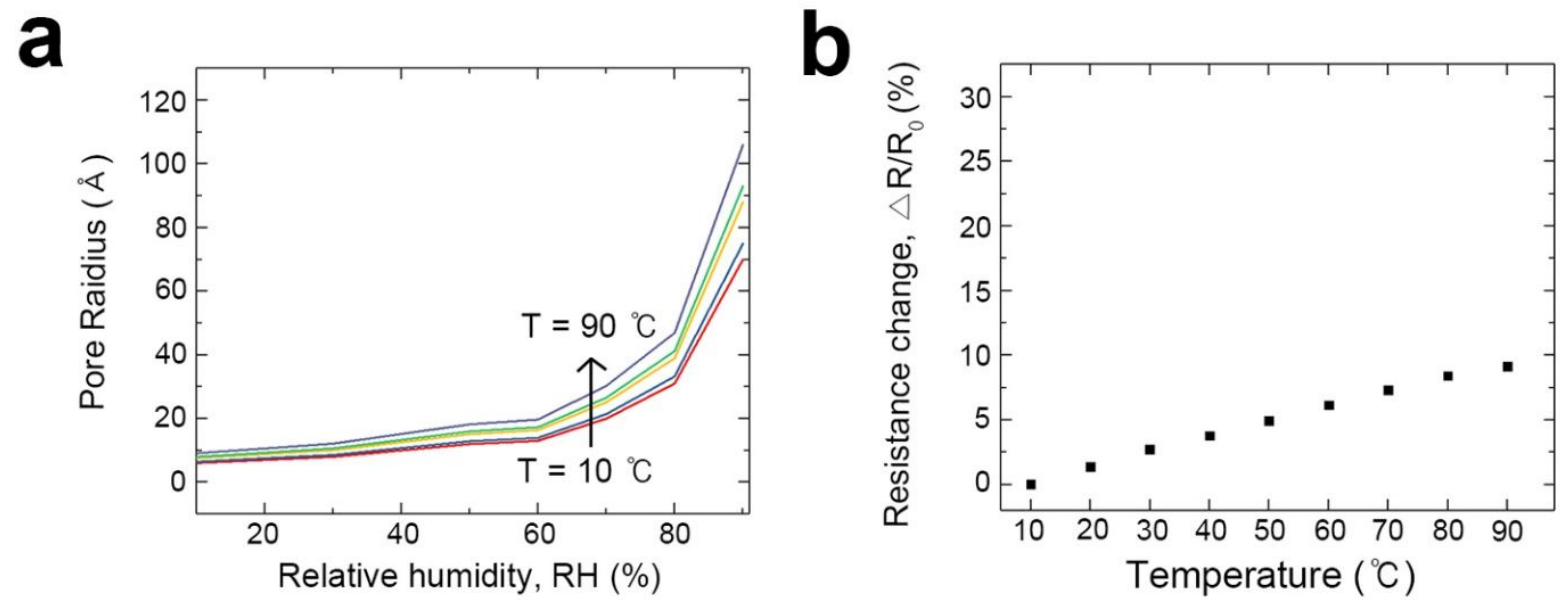

Figure S6. Temperature dependence of the humidity sensor. a) Pore radius of capillary condensation (Kelvin equation). ${ }^{[1]}$ b) Normalized resistance changes in response to temperature at a constant ambient humidity level (50 RH\%).

The variation in the output resistance was calculated as $\Delta \mathrm{R} / R_{0}=\left(R_{\text {final }}-R_{\text {initial }}\right) / R_{\text {initial }}$, where $R_{\text {final }}$ is the resistance at a set temperature value, and $R_{\text {initial }}$ is the resistance at $10{ }^{\circ} \mathrm{C}$ $\mathrm{RH} \%$. 


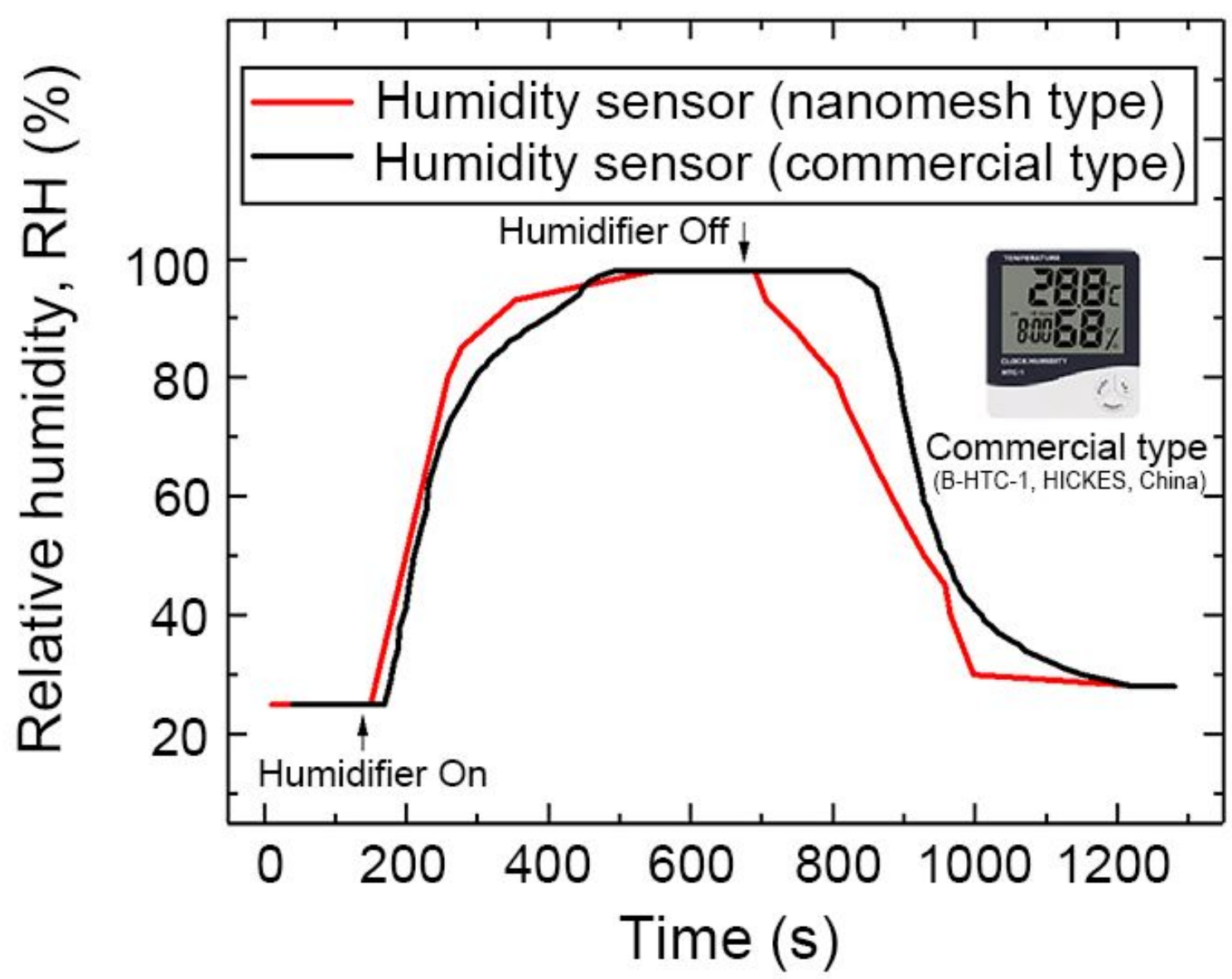

Figure S7. Comparison of response and relaxation times of the nanomesh humidity sensor and a commercially available humidity sensor. 
a

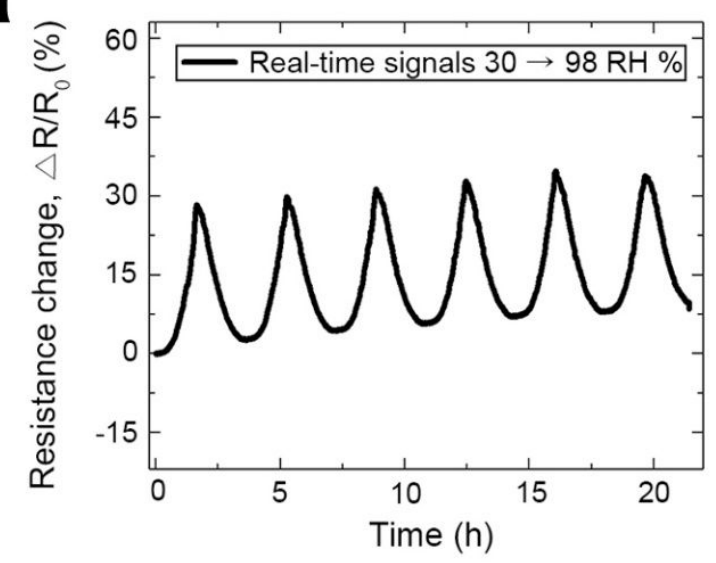

b

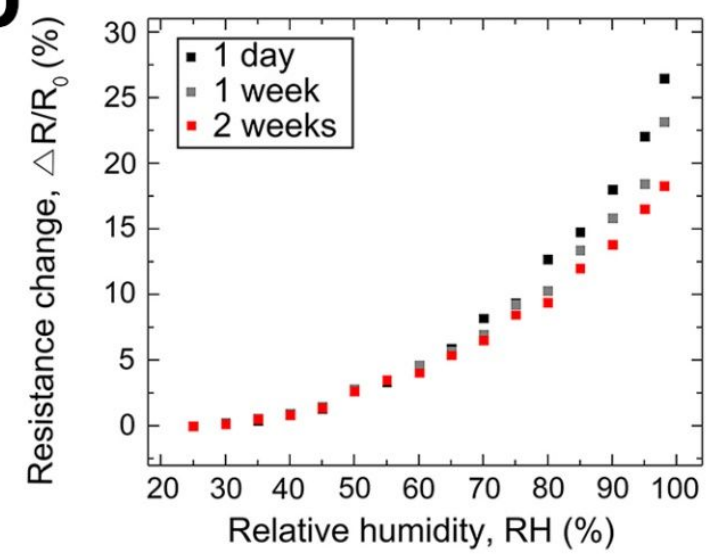

Figure S8. a) Resistance changes of the PVA nanomesh humidity sensor under six cycles ranging from 30 to $98 \mathrm{RH} \%$. b) Long-term stability of the sensor. Resistance change of the sensor with relative humidity after storage for 1 day to 2 weeks, showing the stability of the sensor over time 
a

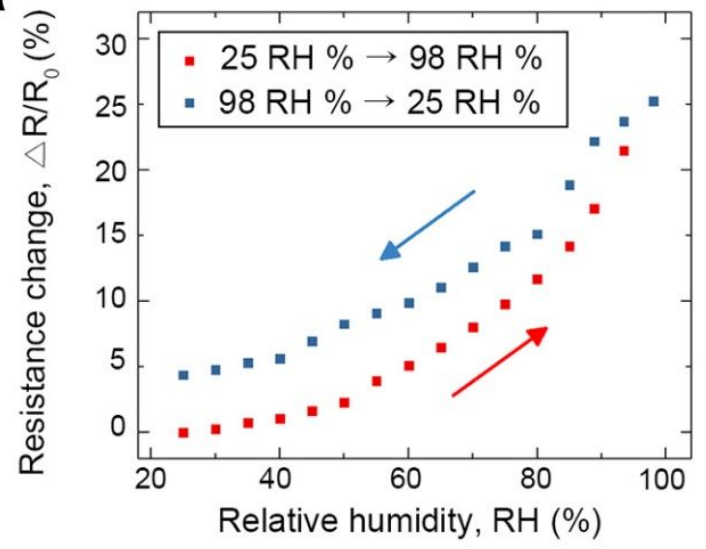

b

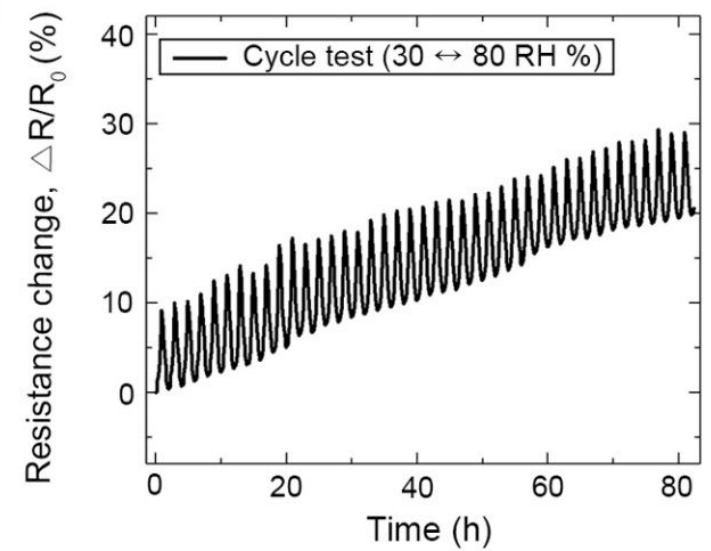

Figure S9. a) Hysteresis characteristic of the sensor, normalized resistance change at intervals of $5 \mathrm{RH} \%$ with increasing (red) and decreasing (blue) humidity. b) Cycle test ranging from 30 to $80 \mathrm{RH} \%$ for 40 cycles. 


\section{Humidity sensor}
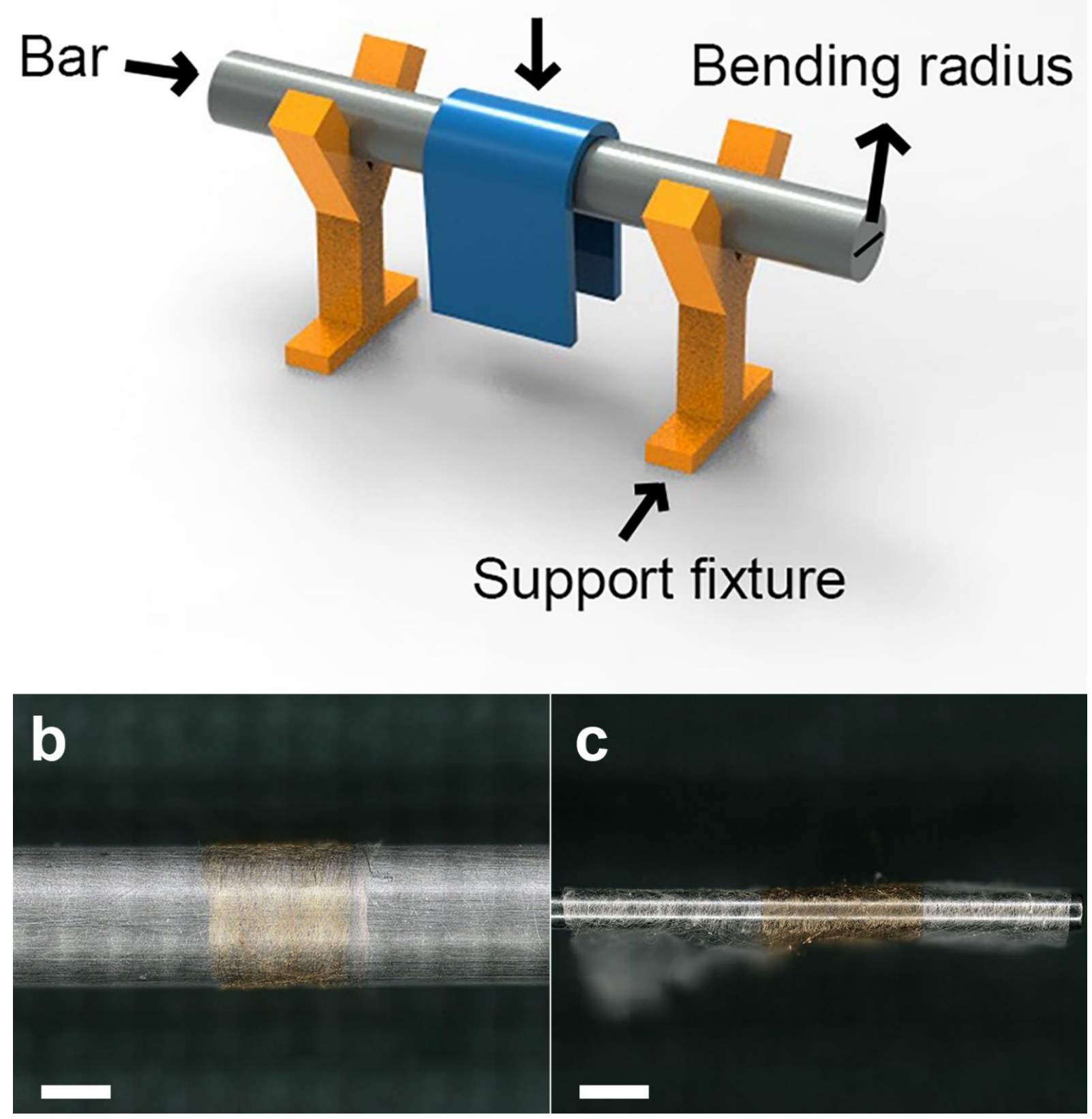

Figure S10. a) Schematic of the flexibility test which was measured in a thermo-hygrostat. Sensor images at bending radius: b) $2 \mathrm{~mm}$ (scale bar $=1 \mathrm{~mm})$. c) $500 \mu \mathrm{m}$ (scale bar $=1 \mathrm{~mm})$ 


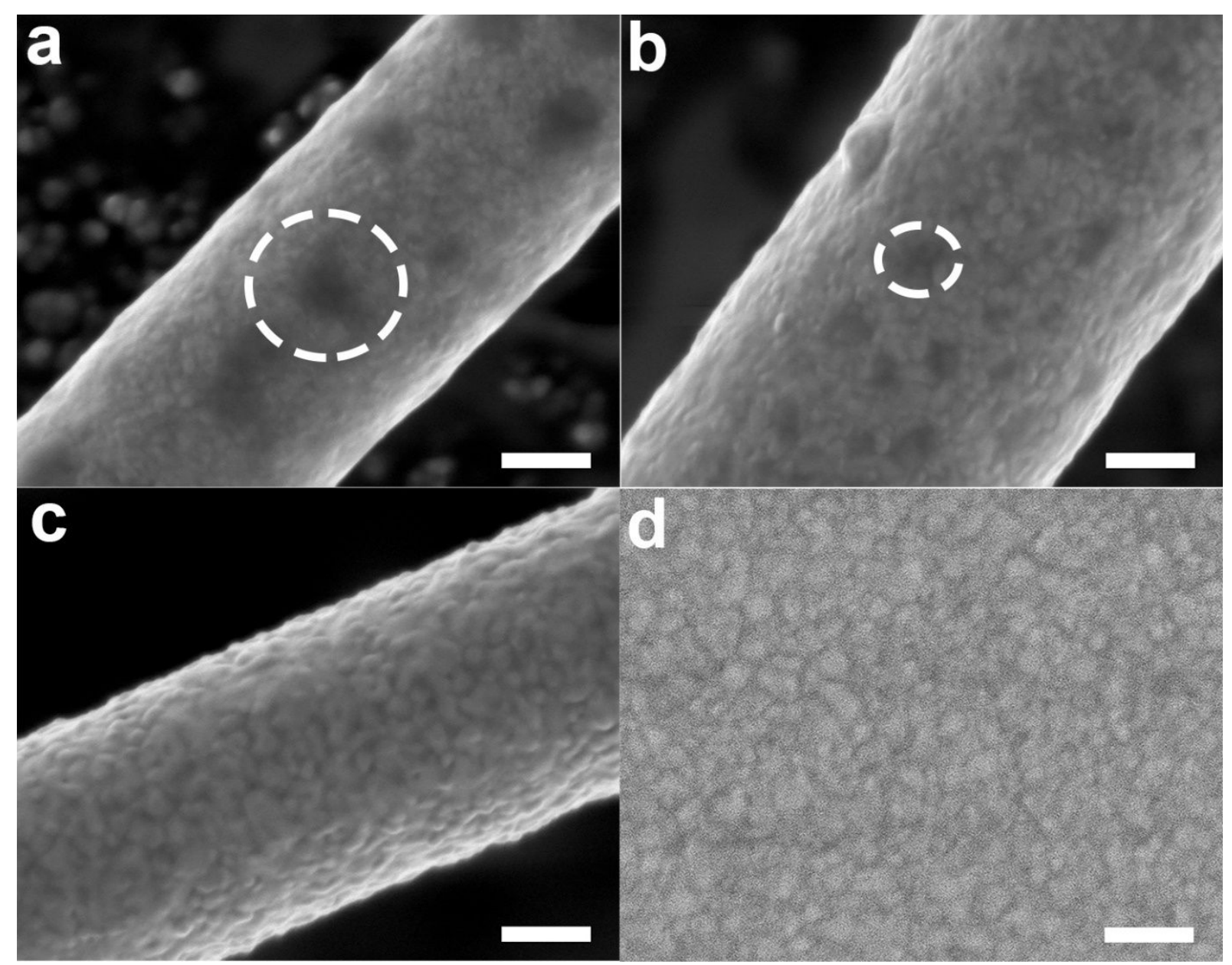

Figure S11. SEM images of gold surface (scale bar = $200 \mathrm{~nm}$ ): a) $100 \mathrm{~nm}$-thick gold coated surface on the PVA nanofiber. b) $200 \mathrm{~nm}$-thick gold coated surfaced on the PVA nanofiber. c) $300 \mathrm{~nm}$-thick gold coated surface on the PVA nanofiber. d) $100 \mathrm{~nm}$-thick gold coated surface on the PVA film.

The first two images of Figure S11 show a discontinuous gold surface. The black dot inside the white circle represents PVA polymer. Figure S11 also shows a continuous gold surface, such as a gold-coated surface on a flat film. With a $\leq 200 \mathrm{~nm}$-thick coating, a discrete gold surface was formed, whereas with a $\geq 300 \mathrm{~nm}$-thick coating, a continuous gold surface was formed. The discrete state of the gold surface causes the resistance change; the sensitivity is also affected by the surface state. 

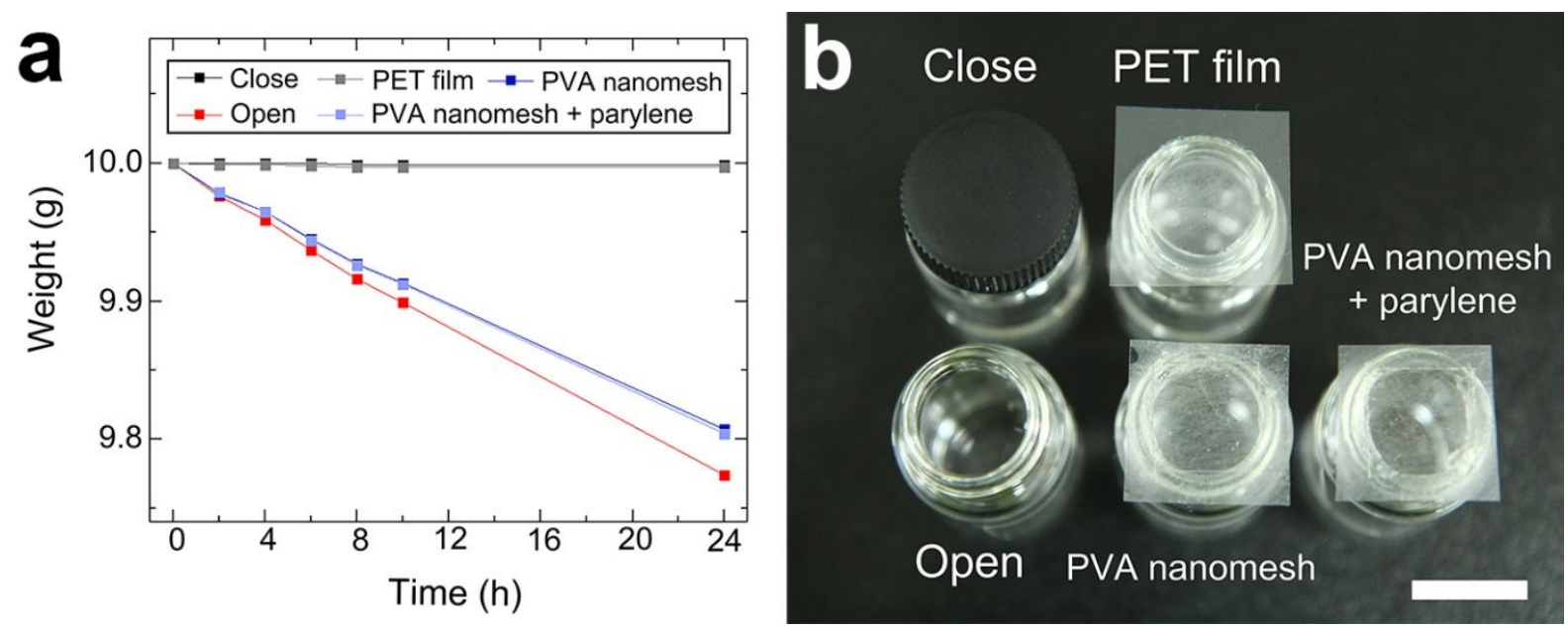

Figure S12. Gas permeability of the sensor: a) Weight of water in a vial over time at each condition. b) Sample images at each condition. (scale bar $=15 \mathrm{~mm}$ ) 


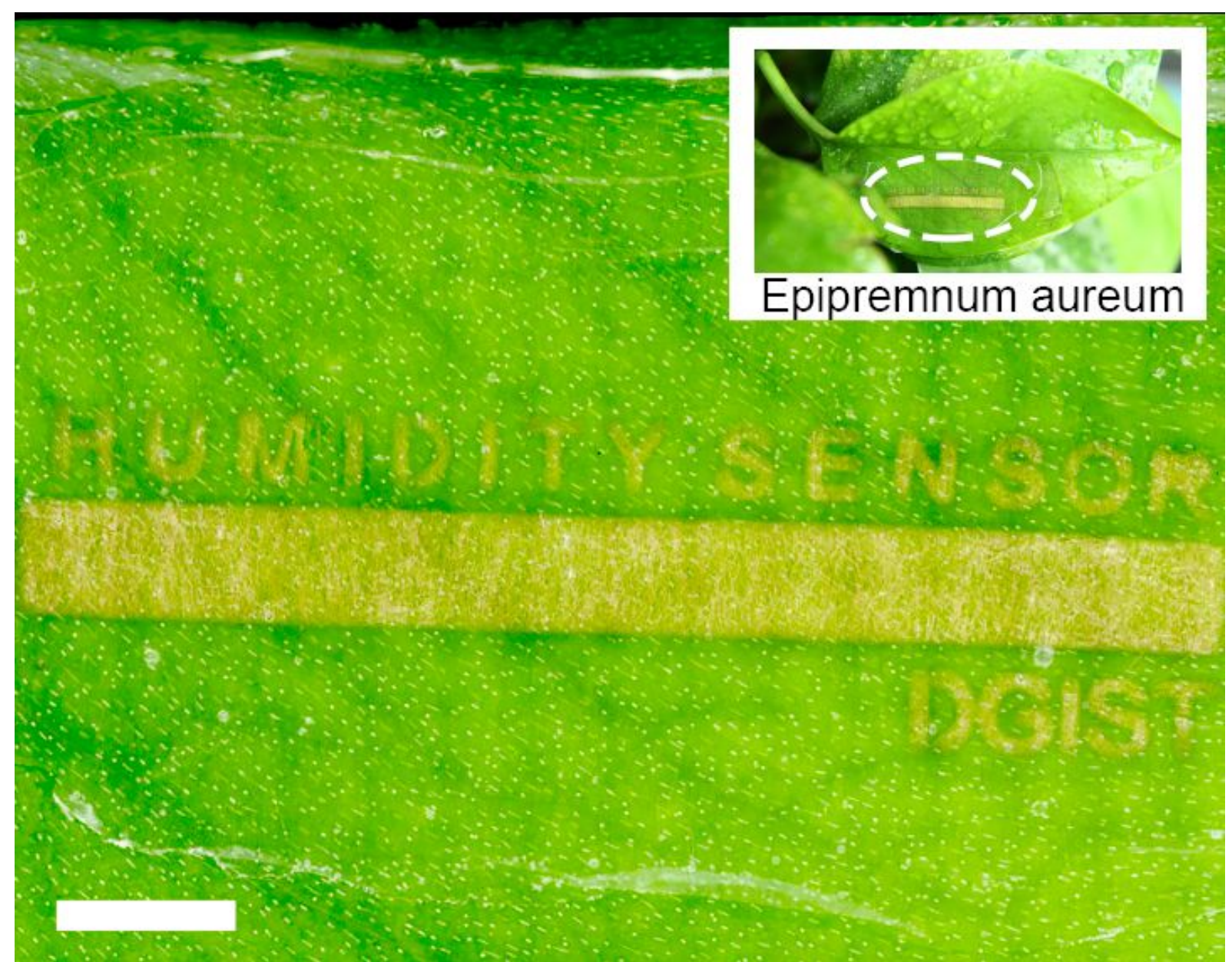

Figure S13. Sensor image attached to the surface of Epipremnum aureum leaf (scale bar $=3$ $\mathrm{mm})$ 


\section{Corresponding Author}

*Corresponding Author. E-mail: swlee@dgist.ac.kr

\section{Reference}

[1] Fratoddi, I.; Bearzotti, A.; Venditti, I.; Cametti, C.; Russo, M. V. Role of nanostructured polymers on the improvement of electrical response-based relative humidity sensors. Sens. Actuators B: Chem. 2016, 225, 96-108. 